\title{
Wald als Umwelt der Informationsgesellschaft
}

\section{Umwelt als Informationsproblem}

Nicht alle, aber viele Umweltprobleme zeichnen sich dadurch aus, daß sie erst einmal erkannt werden müssen. Da die umweltzerstörenden Folgen alltäglicher Handlungsweisen meist zeitlich verzögert oder räumlich getrennt von Handlungen auftreten oder aber ohne besonderes Vorwissen oder ohne besondere Beobachtungsvorkehrungen nicht erkennbar sind, müssen wenige «ExpertInnen» und spezielle Institutionen für die gesamtgesellschaftliche Erkennung und Anerkennung dieses Problems sorgen, bevor in der Bevölkerung oder ihren politischen Organen Handlungsweisen oder Rahmenbedingungen des Handelns verändert werden. Umweltprobleme sind unter diesen Bedingungen immer auch Informationsprobleme, Probleme des Mitteilens, des Verstehens und der politischen Willensbildung.

Am Beispiel der Beziehung der SchweizerInnen zu ihrem Wald werde ich versuchen zu zeigen, welche Bedeutung sozialen Strukturen, Institutionen und Kommunikationsprozessen für die Behandlung der Umwelt und für die Bewältigung von Umweltproblemen zukommt. Im folgenden werde ich auf die Bedeutung des Waldes für die Gesellschaft, den Wandel der Beziehungen zum Wald und die Verarbeitung des "Waldsterbens» in Wissenschaft, Medien und Bevölkerung eingehen. Jede Gesellschaft steht in wechselseitiger Abhängigkeit mit ihrer Umwelt. In der Informationsgesellschaft tritt die Welt den Individuen immer vermittelter entgegen. Der Wald dient mir als Beispiel dafür, wie die Informationsgesellschaft mit ihrer Umwelt umgeht. Meine Ausführungen beziehen sich teilweise auf Befragungsergebnisse aus einem Forschungsprojekt über umweltbezogenes Handeln im Tessin und in der Deutschschweiz (vgl. Tab. 1).'

\section{Vielfalt der Waldbedeutungen}

Der Wald des neuen schweizerischen Waldgesetzes soll der Gesellschaft als naturnaher Lebensraum, als Schutzwald, als Erholungsgebiet und als Holzquelle dienen. ${ }^{2}$ Was sich auf der Ebene des Individuums hinter diesen «Funktionen» verbirgt und daß die offizielle Waldsicht nur als Minimalumschreibung der Bedeutung des Waldes in unserer Gesellschaft anzusehen ist, sollen die folgenden Interviewausschnitte ${ }^{3}$ illustrieren.
ÖkologInnen und UmweltschützerInnen, denen die Funktion «naturnaher Lebensraum» am nächsten liegt, sehen den Wald vielleicht mit ähnlichen Augen wie Monika Wild: «Ich möchte, daß man den Wald als Lebensraum verschiedener Lebewesen, die zusammen leben müssen, begreift. Daß der Wald eben für alle da ist und nicht nur für Jogger oder Hündeler oder nur als Erholungsraum oder nur für die Wildtiere oder die Forstwirtschaft. Ich möchte auch, daß jeder für sich einen Weg im Umgang mit dem Wald sucht, und dazu gehören dann natürlich auch das Waldsterben und die Luftschadstoffe. Für mich ist der Wald der Ort, wo ich das erste Mal und immer wieder wirklich mit Wildtieren in Kontakt gekommen bin, wo ich nicht Hunde und Meerschweinchen, sondern einen Fuchs und einen Dachs gesehen habe. Und natürlich auch die vielen Vogelarten. Das fasziniert mich, und ich bin fürs Leben gern im Wald. Im Wald bin ich von der Natur eingeschlossen, und das gibt mir Geborgenheit.»

Eva Dina, eine Bergbäuerin aus der Leventina, erlebt den Wald hautnah in seiner Schutzfunktion: "Unten im Dorf, wo ich geboren wurde, auf fünfhundert Metern, da sind 1964 Lawinen gleich neben dem Dorf niedergegangen und haben ein schönes Stück Wald zerstört. Die Pflanzen wachsen dort nun von selbst nach, denn es ist nicht wie hier oben, wo sie immer gleich wieder aufforsten. Wir hatten einen schönen Wald oberhalb des Hauses, der jetzt gerodet werden soll. Dort hat es alte Lärchen, die eigentlich den Dorfteil, aus dem ich komme, schützen sollten. Sie werden sie fällen, weil sie ein Projekt für ein Mauerwerk haben, das die Lawinen umleiten soll. 1983, als ich unten war, ist auch eine Lawine runtergegangen und hat Bäume entwurzelt. Man sieht auch, daß dort der Wald zurückgeht.»

Robert Schwarz sucht im Wald auf moderne Weise Erholung. «Der Wald ist für mich eine große Fitneßhalle. Ich gehe regelmäßig viermal in der Woche rennen. Mir ist am Wald sehr wichtig, daß ich mich dort bewegen kann. Im Wald suche ich Ruhe, auch von Autos und so. Ich muß einfach zügig rennen, ohne daß ich stolpere, darum kann ich nicht der Straße entlang rennen. Wenn ich im Wald bin, sind meine Gedanken beim Sport. Da kann ich meine Aggressionen abbauen.»

Wolfgang Zierhofer, Geograph, Geographisches Institut der ETH, Winterthurerstraße 190, 8057 Zürich 


\section{Anlage der Empirie des Forschungsprojektes "Formen umweltbezogenen Handelns..."}

Teil A: Themenzentrierte Interviews:

Je 10 Personen aus den Regionen Bellinzona und

Zürich, die sich nach Alter, Geschlecht, Beruf,

Bildung und politischer Einstellung unterscheiden, wurden zweimal ca. eineinhalb Stunden lang interviewt.
Teil B: Postalische Befragung:

Vorwiegend standardisierter Fragebogen;

Stichproben: Gotthard Südund Gotthard Nord.

Systematische Ziehung von Erwachsenen in Airolo und Biasca bzw. Altdorf und Göschenen; repräsentativ für diese Gemeinden.

Tab. 1

Dem Bauern Daniel Spar erscheint der Wald zuerst einmal als Konglomerat persönlicher Nutzungsmöglichkeiten, bevor er an seine anderen Funktionen denkt: "Selbst besitzen wir keinen Wald, aber als wir diesen Schopf bauten, haben wir Holz gekauft. Der Wald ist natürlich einmal eine Rohstoffreserve, also Brennmaterial oder Baustoff. Aber er produziert auch viel Sauerstoff und beeinflußt das Klima. Für den Wasserhaushalt ist er wichtig. Wir haben eine Quelle für unsere drei, vier Häuser, die aus dem Wald heraus kommt. Natürlich ist er auch ein Erholungsgebiet und ein Lebensraum. Im Berggebiet ist er Lawinenschutz und Erosionsschutz. Es ist schon auch schön, wenn man im Frühling zum Fenster hinaus schaut und es zu grünen beginnt - aber wie soll ich sagen, für uns gehört er einfach zum Alltag. Ich sehe jeden Tag zum Wald hinüber, das ist wie das Land ringsherum oder die Berge oder die Häuser im Dorf unten, oder Morgen und Abend - er gehört einfach dazu.»

Für die meisten befragten Personen sind mehrere Waldfunktionen von Bedeutung, und die vier im Gesetz erwähnten Bedeutungen scheinen in den Interviews in vielfältigen Abwandlungen auf. So deckt beispielsweise die Erholungsfunktion so verschiedene Aktivitäten wie Wandern, die Hunde spazierenführen, Pilze und Beeren sammeln, Vögel beobachten und Lauftraining ab. Für Regula Bach, eine weitere Bäuerin, fällt die Erholungsfunktion geradezu mit der Negation der Nutzenfunktion zusammen, denn für sie ist der Wald zuerst einmal eine Fläche, die nicht von ihr bearbeitet werden muß. Hinter den vier eindeutig und säuberlich getrennt erscheinenden Begriffen, nach denen der Wald in Zukunft bemessen werden soll, verbirgt sich eine Vielfalt individueller Bedeutungen, die sich weder immer widerspruchsfrei fügen noch immer eindeutig zuordnen lassen. Zwischen dem Gesetz und seiner Anwendung liegen Interpretationsschritte, die nicht interessenneutral sein können. Verständigungsbemühungen, Interessensausgleich und eventuell sogar Konfliktlösungsprozesse sind zu erwarten. Dafür werden vielfältige Informationen über den Wald und über die Gesellschaft gefragt sein.

\section{Wald und gesellschaftliche Reproduktion}

Die vier hier erwähnten Waldfunktionen spiegeln so etwas wie das Waldverständnis der gegenwärtigen und offi- ziellen Schweiz. ${ }^{4}$ Sie liegen beispielsweise auch der neuen Konzeption des Landesforstinventars ${ }^{5}$ zugrunde. Auch wenn es heute vielleicht als Maß der Dinge gilt, ist dieses Waldverständnis nur ein heutiges, hiesiges und eines unter vielen. Das mögen die zwei folgenden Interviewausschnitte verdeutlichen:

Mario Niva: "Als wir Kinder waren, hatten wir einen Wald am Monte Ceneri. Unser Kastanienwald war so sauber, daß man auf der Erde essen konnte! Wohin wir auch gegangen sind, da waren das Holz, das Laub, alles geordnet. Und die Kastanien waren unser Leben. Wenn es da keine Kastanien gehabt hätte, wären wir an Hunger gestorben. Der Kastanienwald war die schönste Sache, die es gab. Da waren Bäume, zweihundert, ja dreihundert Jahre alt. Darum war der Wald ein Park. Heute ist nichts mehr von dem. Ich weiß auch nicht, aber da kommen die Mailänder zum Kastaniensammeln. Nicht wir! Das ist schade, sehr schade!»

Karin Frauensteiner: "Ich bin schon immer in den Wald gegangen, früher zum Rennen, jetzt nur noch zum Spazieren und um an die frische Luft zu kommen. Seit etwas mehr als einem Jahr bin ich in einer Gruppe, die die Jahreszeiten mit Ritualen feiert. Also z. B. Winteranfang, wenn das Licht wieder zunimmt. Da bin ich allein in den Wald gegangen, um irgendwo eine Kerze aufzustellen. Einfach so, als Symbol für das Licht, das jetzt wieder kommt. Es geht mir dabei vor allem um die Natur und darum, mit dem zu leben, was uns die Natur zu sagen hat. Dazu gehören z. B. Mondphasen oder auch das Wahrnehmen von alten Bäumen. Ich glaube daran, daß die Bäume Kräfte aussenden. Der Wald ist beruhigend, und manchmal gehe ich in den Wald, weil es mir nicht so gut geht. Dann schaue ich, wie er sich verändert, je nach Jahreszeit, die Vegetation am Boden, die Beleuchtung und die Farben. Er hat für mich eine Qualität, die mir guttut, die ich mitnehme.»

Am Beispiel des Herrn Niva, der den Wald als Nahrungsquelle in Erinnerung hat, erkennen wir, daß mit der gesellschaftlichen Entwicklung der Wald seine Bedeutung ändert. Frühere Volkswirtschaften kannten ganz andere Waldnutzungen, wie z. B. die Eichelmast für Schweine. Bedeutsam für den Wald waren und sind einerseits die Substitutionsmöglichkeiten von Holz als Energiequelle und als Baumaterial. Die fossilen Brennstoffe, v. a. die Kohle für die Schwerindustrie, brachten eine enorme 
Entlastung der Holznutzung. Wegen ihrer klimatologischen Folgeprobleme könnten sie künftig jedoch wieder teilweise durch Holz ersetzt werden. Andererseits verändern sich auch die Kenntnisse im Waldbau, rechtliche Rahmenbedingungen, die Nutzungen der Gewässer und andere, für den Wald relevante Umwelteigenschaften. Nach Baumaltern gemessen, wurde der Wald erst in jüngster Zeit als Erholungsraum und als Reservat der Natur entdeckt. Da der Aufbau des Waldes stark von seiner Nutzung bestimmt wird, verändert sich seine Erscheinungsform parallel zu Formen materieller Reproduktion der Gesellschaft.

Karin Frauensteiner steht als Beispiel für die Bedeutung des Waldes in der ideellen Reproduktion von Gesellschaften. Vielen unserer InterviewpartnerInnen ist der Wald - gleich den Bergen - Inbegriff reiner, romantischer Natur. Wald als Natur ist für unsere InterviewpartnerInnen nicht nur ein Rahmen für Freizeitaktivitäten, sondern neigt auch zur Transzendenz: Im Wald ist der Mensch von Natur umgeben, in ihr geborgen oder verloren; am Wald muß der Mensch Maß nehmen, in ihm offenbart sich die Schöpfung. Heute schleppen jedoch viele das Bewußtsein von Verschmutzung, Raubbau und Vernachlässigung von Wald und Natur im Horizont ihrer Gedanken mit. Die einstige Unbeschwertheit dem Wald gegenüber mag sich nicht mehr einstellen. Folgt man unseren Interviews, so leben wir in einer Zeit, in der der Wald nicht wie in Märchen den Bannkreis des Bösen und Fremden abgibt, sondern uns den Spiegel des Bösen und Fremden in uns selbst vorhält. Durch die ökologische Bedrohung wird der Mythos vom Wald als reiner Natur noch gesteigert.

Während die materielle Reproduktion der Industrie- und Informationsgesellschaft die ihr gemäßen Waldformen ${ }^{6}$ hervorbringt, führt auch die ideelle Reproduktion zu einem besonderen Wissen vom Wald, das Ideologie ist, soweit es die Herrschaftsverhältnisse über den Wald leugnet.

\section{Paradigmenwechsel}

Dem «Waldsterben» dürften wir einen beschleunigten Wandel des Verständnisses von Wald in unserer Gesellschaft verdanken. Gegenüber der einst dominierenden forstwirtschaftlichen Sicht des Waldes gewinnen ökologische Argumente an Geltung. Auch gesamtgesellschaftlich zeichnet sich ein Perspektivenwechsel von Ökonomie zu Ökologie ab; deutlich an Wohlstandsskepsis und politischer Rhetorik zu erkennen, aber erst in Ansätzen verwirklicht. So läßt sich der Wandel der Waldbedeutung als Teil eines umfassenden kulturellen Rationalisierungsprozesses verstehen, der seinen Treibstoff neben dem «Waldsterben» noch aus einer Reihe weiterer Quellen bezieht.

Für den Wald bedeutet dieser Paradigmenwechsel wahrscheinlich folgendes: langfristig werden sich die Waldnutzungen noch stärker an den Waldfunktionen (Schutz,
Erholung, Holz) ausrichten, darüber hinaus werden ungenutzte Waldreservate ${ }^{7}$ entstehen und große Teile des genutzten Waldes naturnäher gehalten; die Zuständigkeiten und Einflußnahmen auf den Wald werden sich etwas wandeln, indem sich die Forstwirtschaft den Anliegen des Naturschutzes öffnet, sich aber zugleich politisch gegen Risiken für den Wald, wie z. B. die Immissionen und den hohen Wildbestand, wehrt ${ }^{8}$; der Wald wird fortan mit "anderen Augen», nämlich durch ein Waldbeobachtungssystem gesehen werden.

\section{Beobachtungsprobleme}

Wie es mit der Gesundheit der Waldbäume weitergehen wird, muß trotz weltweiter intensiver Forschungen noch immer Spekulation bleiben. Freilich gab und gibt es Stimmen, die schon immer darüber Bescheid wußten. Doch setzt man wissenschaftliche Rationalität als Maßstab, muß man sich vorläufig noch langfristiger Prognosen enthalten und Wissensnotstand erklären. In der Schweiz wird zwar seit 1984 eine Zunahme von Nadel- oder Blattverlusten beobachtet. Eine erhöhte Sterberate der Bäume läßt sich jedoch nicht feststellen. Der Beobachtungszeitraum gilt immer noch als sehr kurz für verläßliche Trendaussagen, und ungeklärt ist auch die natürliche Variabilität der Kronenverlichtung. ${ }^{9}$ Werden Anzeichen von Schwäche beobachtet, oder sind die «neuartigen Waldschäden» nur eine Täuschung?

Als ab 1982 das "Waldsterben» massenmedial verbreitet wurde, gingen offizielle Dokumente meist vom Faktum der Waldschäden und von einem offensichtlichen $\mathrm{Zu}$ sammenhang mit der Luftverschmutzung aus. ${ }^{10}$ Vor dieser Folie mußten einige Jahre später die Erkenntnis, daß der Wald nicht im Sterben liegt, und die Besinnung auf das Ideal der Objektivität als politisches Zurückkrebsen der Wissenschaft erscheinen und kritisiert werden."

\section{Hat der Wald noch Zukunft?}

Wie war das damals 1982/83, als wir zum ersten Mal aus den Medien von den Schäden im Wald erfuhren? Getreu den Bildern aus den Medien wurde das Waldsterben von einigen unserer Interviewten sehr wörtlich, ähnlich wie von Regula Bach, aufgefaßt: "Das hat mich wahnsinnig erschreckt und betroffen gemacht: Die Vorstellung, daß es tatsächlich im Bereich des Möglichen liegt, daß der Wald eines Tages - gut, es wird vielleicht noch Büsche geben - nicht mehr sein könnte, daß das eine reelle Möglichkeit ist, daß ihn meine Kinder nicht mehr so erleben könnten! Damals habe ich mir vorgestellt, daß es eben wirklich in ein paar Jahren schon den Wald um unser Haus herum nicht mehr geben könnte.»

Der Wald steht noch. Es ist stiller um das Waldsterben geworden, ohne daß so etwas wie eine offizielle und endgültige Entwarnung in dieser Angelegenheit gekommen wäre. Man wartet. Aber worauf? Wir wollten von unseren schriftlich Befragten wissen, welchen Waldzustand sie 
für das Jahr 2000 erwarten, falls keine weiteren Maßnahmen zum Schutz des Waldes ergriffen würden (vgl. Tab. 2). ${ }^{12}$ Bei den UrnerInnen wie bei den TessinerInnen erwartet fast niemand eine Verbesserung, aber mehr als drei Viertel glauben, daß es ohne weitere Maßnahmen dem Wald schlechter gehen würde!

\begin{tabular}{|c|c|c|c|c|c|}
\hline \multicolumn{6}{|c|}{$\begin{array}{l}\text { Stellen Sie sich vor, es würden keine zusätzlichen } \\
\text { Massnahmen zum Schutz des Waldes ergriffen: } \\
\text { Was glauben Sie, wie wird der Waldzustand im } \\
\text { Jahr } 2000 \text { im Vergleich mit heute sein? }\end{array}$} \\
\hline Antworten & \multicolumn{4}{|c|}{ Gewichtete Prozentwerte } & \multirow[b]{2}{*}{$\begin{array}{l}\text { TOTAL } \\
(n=1191)\end{array}$} \\
\hline & Besser & $\begin{array}{l}\text { Etwa } \\
\text { gleich }\end{array}$ & $\begin{array}{c}\text { Schlech- } \\
\text { ter }\end{array}$ & TOTAL & \\
\hline $\begin{array}{l}\text { Gotthard Nord } \\
\text { Gotthard Süd }\end{array}$ & $\begin{array}{l}1.71 \\
2.69\end{array}$ & $\begin{array}{l}20.96 \\
20.08\end{array}$ & $\begin{array}{l}77.33 \\
77.24\end{array}$ & $\begin{array}{l}100.00 \\
100.00\end{array}$ & $\begin{array}{l}201 \\
179\end{array}$ \\
\hline
\end{tabular}

Tab. 2

Aus den Interviews werden die Zahlen verständlicher. Es herrscht ein seltsam unbestimmter Pessimismus, denn viele hegen zwar Befürchtungen, können aber nur sagen, daß es sich anders zutragen wird, als sie es sich früher vorgestellt hatten. So beispielsweise Andreas Ruh: "Also ich stelle mir im Moment keinen großflächigen Ökokollaps vor. Das wird zwar wieder relativiert bei diesen Stürmen. Es hat mich sehr beeindruckt, als ich da in Medels war und die Wälder gesehen habe, wo es quadratkilometerweise einfach, tack, tack, tack, jeden Baum umgeschletzt hat. Lokal kann es schon zu derartigen Ökokollapsen kommen, aber für die Schweiz und ihren Wald... wenn ich so ganz kurz darüber nachdenke... es wird zwar etwas gegen die Schadstoffe unternommen, doch der Schadstoffaussto $\beta$ wächst trotzdem weiter, und die Wälder sind immer noch krank. Irgendwie hat man jetzt das Gefühl, es habe sich stabilisiert. Und mindestens so der erste Schub Bewußtsein, Staunen, oder wie man dem auch sagen will, ist vorüber. In meinem Kopf geistert nicht die Vorstellung herum, daß in zehn Jahren die Wälder alle nur noch dürr und tot dastehen. Gesamtschweizerisch glaube ich nicht, daß so etwas passieren wird.”

\section{Wer trägt die Schuld?}

In beiden Stichproben gilt die Luftverschmutzung als Hauptursache der Waldschäden (vgl. Tab. 3). Andere Einflußfaktoren werden mit Abstand als unbedeutender beurteilt. In der Leventina wird der unsachgemäßen Waldpflege und dem Wetter mehr Einfluß beigemessen, im Reußtal eher den Fehlern bei der Aufforstung. Offensichtlich genießt die Forstwirtschaft, die sich selbst gerne als die Hegeinstanz des Waldes begreift ${ }^{13}$, nicht mehr das volle Vertrauen der Bevölkerung.

Die Frage nach den Ursachen ist zugleich die Frage nach den biologischen und physikalischen Kausalzusammenhängen. Sind letztere bekannt, lassen sich die Verursacher bestimmen, moralisch anklagen sowie gegebenenfalls rechtlich oder politisch zur Verantwortung ziehen. Die Kenntnis von Kausalzusammenhängen ist - neben konsensfähigen Zielen - auch der wichtigste Faktor zur Begründung von Gegenmaßnahmen. Somit wird die Frage der Kausalitäten zum eigentlichen Angelpunkt zwischen Naturwissenschaft und Politik! Gerade diese Frage ist heute aber offener denn je.

Wir simulierten in unserem Fragebogen auch eine Entscheidungssituation mit zugleich hohem Risiko und Wissensnotstand, wie sie für das "Waldsterben» typisch ist (vgl. Tab. 4). In beiden Stichproben sprach sich eine große Mehrheit dafür aus, das Umweltrisiko zulasten wirtschaftlicher Risiken zu mindern. Da die Realität des Fragebogens viele Faktoren nicht berücksichtigt, die bei politischen Entscheiden durchaus eine Rolle spielen, läßt sich nur sagen, daß eine progressive Umweltpolitik argumentativ verstanden wird, aber nicht, daß sie akzeptiert würde.

\begin{tabular}{|c|c|c|c|c|c|c|c|}
\hline Wie hoch schätzen Sie den Anteil der folgenden Einfluss & & dan. & lophä & CnMor & Ananging & & \\
\hline & & & Gewicht & e Prozen & werte & & \\
\hline $\begin{aligned} \mathrm{GN} & =\text { Gottha } \\
\mathrm{GS} & =\text { Gotthe }\end{aligned}$ & & $\begin{array}{l}\text { Hoher } \\
\text { Anteil }\end{array}$ & $\begin{array}{l}\text { Tiefer } \\
\text { Anteil }\end{array}$ & $\begin{array}{l}\text { Keinen } \\
\text { Anteil }\end{array}$ & \begin{tabular}{|c|} 
Das weiss \\
ich nicht
\end{tabular} & TOTAL & $\begin{array}{c}\text { TOTAL } \\
(n=1191)\end{array}$ \\
\hline Luftverschmutzung durch den motorisierten Verkehr & $\begin{array}{l}\text { GN } \\
\text { GS } \\
\end{array}$ & $\begin{array}{l}72.87 \\
67.42\end{array}$ & $\begin{array}{l}22.96 \\
28.72\end{array}$ & $\begin{array}{l}1.59 \\
2.96 \\
\end{array}$ & $\begin{array}{l}2.57 \\
0.90 \\
\end{array}$ & $\begin{array}{l}100.00 \\
100.00 \\
\end{array}$ & $\begin{array}{l}204 \\
184 \\
\end{array}$ \\
\hline Luftverschmutzung durch Gebäudeheizungen und Industrie & $\begin{array}{l}\text { GN } \\
\text { GS } \\
\end{array}$ & $\begin{array}{l}84.81 \\
85.16 \\
\end{array}$ & $\begin{array}{l}12.79 \\
13.63\end{array}$ & $\begin{array}{l}0.80 \\
0.30\end{array}$ & $\begin{array}{l}1.60 \\
0.91\end{array}$ & $\begin{array}{l}100.00 \\
100.00\end{array}$ & $\begin{array}{l}203 \\
184 \\
\end{array}$ \\
\hline Das harte Wetter der letzten zehn Jahre & $\begin{array}{l}\text { GN } \\
\text { GS }\end{array}$ & $\begin{array}{l}19.97 \\
27.31\end{array}$ & $\begin{array}{l}40.87 \\
48.34\end{array}$ & $\begin{array}{l}31.28 \\
16.01\end{array}$ & $\begin{array}{l}7.88 \\
8.34\end{array}$ & $\begin{array}{l}100.00 \\
100.00\end{array}$ & $\begin{array}{l}202 \\
181\end{array}$ \\
\hline Eine unsachgemässe Waldpflege und Waldbewirtschaftung & $\begin{array}{l}\text { GN } \\
\text { GS } \\
\end{array}$ & $\begin{array}{l}39.79 \\
54.58\end{array}$ & $\begin{array}{l}36.34 \\
33.27\end{array}$ & $\begin{array}{r}15.35 \\
8.00\end{array}$ & $\begin{array}{l}8.52 \\
4.16 \\
\end{array}$ & $\begin{array}{l}100.00 \\
100.00\end{array}$ & $\begin{array}{l}201 \\
183 \\
\end{array}$ \\
\hline Fehler bei der Aufforstung in der Vergangenheit & $\begin{array}{l}\text { GN } \\
\text { GS }\end{array}$ & $\begin{array}{l}39.58 \\
26.14\end{array}$ & $\begin{array}{l}30.26 \\
39.70\end{array}$ & $\begin{array}{l}15.12 \\
14.87\end{array}$ & $\begin{array}{l}15.04 \\
19.29\end{array}$ & $\begin{array}{l}100.00 \\
100.00\end{array}$ & $\begin{array}{l}201 \\
183 \\
\end{array}$ \\
\hline
\end{tabular}

Tab. 3 


\begin{tabular}{|c|c|c|c|c|}
\hline \multirow{2}{*}{\multicolumn{5}{|c|}{$\begin{array}{l}\text { Wie sollten Ihrer Meinung nach die Politiker auf die } \\
\text { folgende Situation reagieren? } \\
\text { Situation: Eine Substanz gelangt in grossen Mengen in die Luft. } \\
\text { Man vermutet, dass dadurch Lebewesen geschädigt werden. } \\
\text { Die Wissenschaft konnte bis jetzt weder die Schädlichkeit, noch } \\
\text { die Unschädlichkeit nachweisen. Es wird weiter geforscht. Ein } \\
\text { Verzicht auf die Substanz würde Arbeitsplätze kosten und viele } \\
\text { Güter verteuern. } \\
\text { Wie sollten die Politiker Ihrer Meinung nach reagieren? }\end{array}$}} \\
\hline & & & & \\
\hline \multicolumn{2}{|c|}{$\begin{array}{l}\text { Reaktion A: } \\
\text { Solange man nicht wirklich } \\
\text { weiss, ob die Substanz } \\
\text { schädlich ist, sollte man sie } \\
\text { nicht verbieten. }\end{array}$} & \multicolumn{3}{|c|}{$\begin{array}{l}\text { Reaktion B: } \\
\text { Solange man nicht wirklich } \\
\text { weiss, ob die Substanz } \\
\text { schädlich ist, soltte man sie } \\
\text { verbieten. }\end{array}$} \\
\hline & \multicolumn{3}{|c|}{ Gewichtete Prozentwerte } & \multirow{2}{*}{$\begin{array}{l}\text { TOTAL } \\
(n=1191)\end{array}$} \\
\hline & Reaktion A & Reaktion B & TOTAL & \\
\hline $\begin{array}{l}\text { Gotthard Nord } \\
\text { Gotthard Süd }\end{array}$ & $\begin{array}{l}22.43 \\
15.39\end{array}$ & & & $\begin{array}{l}199 \\
178\end{array}$ \\
\hline
\end{tabular}

Tab. 4

\section{Alles manipuliert?}

Urteile der Laien über das Waldsterben beruhen im wesentlichen auf massenmedial vermittelten und quasi industriell verbreiteten Informationen. Dieses Abhängigkeitsverhältnis ist nicht ohne weiteres auch ein Vertrauensverhältnis: "Alle Informationen, die sie geben, sind derart widersprïchlich. Und ich bin auch wissenschafilich nicht kompetent. Mir können sie vieles sagen. Aber trotzdem: Zwei und zwei kann man schon noch zusammenzählen, nicht wahr. Und dann bemerkt man, daß da Dinge behauptet werden, die sicher nicht möglich sind! Ich glaube, es wird gefiltert. (...) Die Informationen werden manipuliert, um die Bevölkerung nicht zu sehr zu erschrecken, um bestimmte Wirtschaftsbereiche nicht zu treffen, z. B. den Tourismus. Davon bin ich zutiefst überzeugt», argwöhnt Anna Ruffini. Mit der Berichterstattung über den Wald und die Waldschäden ist in beiden Stichproben die Mehrheit der Befragten nicht zufrieden (vgl. Tab. 5)! Der Anteil Unzufriedener ist jedoch im Tessin wesentlich höher. Die TessinerInnen beklagen in erster Linie, daß nicht ausreichend über den Wald berichtet wird. Den UrnerInnen sind dagegen die Meldungen vor allem zu widersprüchlich. Ihnen erscheinen die Berichte aber eher glaubwürdig als den TessinerInnen. Am seltensten wird in beiden Stichproben bemängelt, daß die Waldschäden übertrieben werden. Die Unzufriedenheit scheint damit im Norden eher auf die massenmedial ausgetragenen politischen Auseinandersetzungen zurückzugehen, während sie im Süden am ehesten einer Unterversorgung mit subjektiv sinnvollen Informationen entspringt.

Auch einige unserer InterviewpartnerInnen stehen den Meldungen über den Waldzustand recht skeptisch gegenüber. Bezweifelt wird weniger die Richtigkeit von Prozentzahlen, sondern deren Aussagekraft. Was heißt normal, was heißt stabilisiert, was heißt krank? Was darf man aus den Veränderungen schließen und was nicht? Andere äußern den Verdacht, daß das Thema politisch vereinnahmt werde: Das Waldsterben werde nur dazu benutzt, die AutofahrerInnen zu schikanieren, und die beteiligten Institutionen, z. B. das BUWAL, würden nur Wasser auf die eigenen Mühlen gießen.

\begin{tabular}{|c|c|c|c|c|c|c|}
\hline \multicolumn{7}{|c|}{$\begin{array}{l}\text { Uns interessiert Ihre Meinung zu den Berichten über den Wald in Zeitungen, Radio und Fernsehen. } \\
\text { Können Sie den folgenden Aussagen zustimmen? }\end{array}$} \\
\hline \multirow{2}{*}{\multicolumn{2}{|c|}{$\begin{array}{l}\text { Fragen/ } \\
\text { Stichproben }\end{array}$}} & \multicolumn{4}{|c|}{ Gewichtete Prozentwerte } & \multirow{3}{*}{\begin{tabular}{|r} 
TOTAL \\
$(\mathrm{n}=1191)$ \\
202 \\
182
\end{tabular}} \\
\hline & & \multirow{2}{*}{\begin{tabular}{|l|}
$\mathrm{Ja}$ \\
\\
75.93 \\
57.82 \\
\end{tabular}} & \multirow{2}{*}{\begin{tabular}{|c|} 
Nein \\
\\
12.88 \\
27.31 \\
\end{tabular}} & \multirow{2}{*}{\begin{tabular}{|l|}
$\begin{array}{l}\text { Dazu } \\
\text { kann ich } \\
\text { nichts } \\
\text { sagen }\end{array}$ \\
11.18 \\
14.87
\end{tabular}} & \multirow{2}{*}{\begin{tabular}{|l|} 
TOTAL \\
100.00 \\
100.00 \\
\end{tabular}} & \\
\hline Ich finde die Meldungen über die Waldschäden oft widersprüchlich. & $\begin{array}{l}\mathrm{GN} \\
\mathrm{GS}\end{array}$ & & & & & \\
\hline Die Berichte über die Waldschäden sind manchmal schwer verständlich. & $\begin{array}{l}\text { GN } \\
\text { GS }\end{array}$ & $\begin{array}{l}45.04 \\
55.70\end{array}$ & $\begin{array}{l}44.76 \\
28.55 \\
\end{array}$ & \begin{tabular}{|l|}
10.20 \\
15.76 \\
\end{tabular} & $\begin{array}{l}100.00 \\
100.00\end{array}$ & $\begin{array}{l}203 \\
182\end{array}$ \\
\hline Die Aussagen sind oft durch einen politischen Standpunkt verzert. & GN & $\begin{array}{l}66.67 \\
60.38\end{array}$ & $\begin{array}{l}17.78 \\
11.85\end{array}$ & $\begin{array}{l}15.56 \\
27.77\end{array}$ & $\begin{array}{l}100.00 \\
100.00\end{array}$ & 202 \\
\hline Die Nachrichten über den Wald erscheinen mir glaubwürdig. & $\begin{array}{l}G N \\
G S\end{array}$ & $\begin{array}{l}60.05 \\
45.46\end{array}$ & $\begin{array}{l}29.74 \\
37.94\end{array}$ & $\begin{array}{l}10.21 \\
16.60\end{array}$ & $\begin{array}{l}100.00 \\
100.00\end{array}$ & $\begin{array}{l}200 \\
180\end{array}$ \\
\hline Über den Wald und seinen Zustand wird ausreichend oft berichtet. & $\begin{array}{l}G N \\
G S\end{array}$ & $\begin{array}{l}42.68 \\
22.59\end{array}$ & $\begin{array}{l}52.17 \\
70.18\end{array}$ & $\begin{array}{l}5.14 \\
7.23\end{array}$ & $\begin{array}{l}100.00 \\
100.00\end{array}$ & $\begin{array}{l}204 \\
184\end{array}$ \\
\hline Die Medien tendieren dazu, die Folgen der Waldschäden zu übertreiben. & $\begin{array}{l}\mathrm{GN} \\
\mathrm{GS}\end{array}$ & $\begin{array}{l}29.09 \\
21.20\end{array}$ & $\begin{array}{l}58.94 \\
61.00\end{array}$ & $\begin{array}{l}11.97 \\
17.80\end{array}$ & \begin{tabular}{|l|}
100.00 \\
100.00 \\
\end{tabular} & $\begin{array}{l}204 \\
179\end{array}$ \\
\hline Ich bin insgesamt mit der Berichterstattung über den Wald zufn & $\begin{array}{l}G N \\
\text { GS }\end{array}$ & $\begin{array}{l}41.63 \\
22.72\end{array}$ & $\begin{array}{r}50.85 \\
56.82 \\
\end{array}$ & $\begin{array}{r}7.52 \\
20.46 \\
\end{array}$ & \begin{tabular}{|l|}
100.00 \\
100.00 \\
\end{tabular} & $\begin{array}{l}199 \\
176 \\
\end{array}$ \\
\hline
\end{tabular}

Tab. 5 


\section{Orientierungsbemühungen \\ in der Informationsgesellschaft}

Gemeinhin wird die gesellschaftliche Entwicklung der Moderne in den Sozialwissenschaften als funktionale Differenzierung und als Rationalisierungsproze $\beta$ beschrieben. Funktionale Differenzierung bezeichnet die Spezialisierung und Koordination von Handlungsbereichen, wie Wirtschaft, Verwaltung, Staat, Erziehungswesen, Wissenschaft usw. Im Laufe von Rationalisierungsprozessen verändern sich Auffassungen und damit die Gründe, die für oder gegen bestimmte Handlungsweisen und gesellschaftliche Strukturen angeführt werden. Damit ist auch die Säkularisierung unseres Weltbildes und die Relativierung unserer Kultur und ihrer Werte gemeint.

Funktionale Differenzierung steigert die Effizienz menschlicher Arbeit in physikalischer Hinsicht ungeheuer - wir versetzen Berge. Zugleich kommt den Individuen aber der Einblick und der Einfluß in viele Handlungsbereiche abhanden. Die Welt wird immer mittelbarer, sowohl was Materialströme und Versorgung als auch was die Kommunikation betrifft. Denn Face-to-faceInteraktionen und Primärerfahrungen werden in der Informationsgesellschaft häufig durch Medien aller Art, wie Massenmedien, Geld, Computersysteme und andere Artefakte, ersetzt.

Gegen den Verlust autochthoner Erfahrung baut die Gesellschaft Vermittlungsinstanzen auf: Beobachtungsprogramme, ExpertInnen, Informationskanäle usw. Im Bemühen, eine intime Beziehung zum Wald aufrechtzuerhalten, wurden erst forstwirtschaftliche Planungsinstrumente erfunden, dann wurde die Forstwissenschaft institutionalisiert, und jüngst entstanden die Beobachtungsprogramme "Landesforstinventar» und "Waldschadeninventur", die schon wieder veränderten Informationsbedürfnissen angepaßt wurden.

Auf Folgeprobleme funktionaler Differenzierung wird mit funktionaler Differenzierung geantwortet. Zuständigkeiten werden neu definiert und umverteilt. Wie im Falle des "Waldsterbens» ergeben sich gelegentlich Probleme, die verschiedene Bereiche der Gesellschaft in Resonanz versetzen. Das Problem schwappt sozusagen von der Forstwissenschaft in Politik ${ }^{14}$, Ethik ${ }^{15}$, Recht ${ }^{16}$, Wirtschaftswissenschaften ${ }^{17}$ und in andere Bereiche ${ }^{18}$ über. Zwischen diesen Handlungsbereichen vermitteln meist Fachmedien und spezifische Informationskanäle. Debatten von ExpertInnen, wie etwa die Auseinandersetzungen um Tempolimits zur Luftreinhaltung, finden meist nicht originär in den Massenmedien statt. Letztere produzieren vielmehr die Informationsvermittlung zur Bevölkerung im allgemeinen, vorwiegend nach Maßgabe marktwirtschaftlicher Kriterien. ${ }^{19}$ So wird die einst individuumsnahe Umweltwahrnehmung heute vorwiegend gesellschaftlich organisiert.

Schon in der funktionalen Differenzierung scheint eine Rationalität auf, die sich zunehmend Geltung auf Kosten traditioneller Weltdeutungen und Wertesysteme ver- schafft. Gegen moralischen Relativismus und mediatisierte Welt bieten auch körperliche Berührungen und Wärme - sozusagen instinktive Instanzen - keine versichernden Erfahrungen mehr! Angesichts der globalen Verkettungen von Luftverschmutzung und Weltmarkt hilft es wenig, Bäume im Wald zu umarmen: Treibhauseffekt und Vernichtung der Regenwälder lassen uns «unberührt». Bilder aus den Medien können eigenes Erleben kaum ersetzen. Unsere Moral wird daher vermehrt an Abstraktionen anknüpfen und die Philosophie nach Ethiken für die technische Zivilisation suchen müssen.

\section{Wald und Informationsgesellschaft}

Die Mehrzahl unserer InterviewpartnerInnen schätzt zwar den Wald als wertvolle Umwelt. Sie begreifen ihn allerdings als etwas jenseits von Gesellschaft. Ohne die alltägliche Erfahrung der Verbundenheit, die etwa Mario Niva zu seinem Kastanienwald empfindet, wird der Wald allzu leicht als «Natur» stilisiert und als solche der Gesellschaft gegenübergestellt. So wird der Blick auf wechselseitige Abhängigkeit und Einflüsse zwischen Gesellschaft und Wald verstellt.

Umweltprobleme können aber nur entstehen, wenn die Interdependenzen zwischen materieller und ideeller Reproduktion der Gesellschaft einerseits und die Erscheinungsformen unserer Umwelt andererseits nicht erkannt oder ignoriert werden. Je vermittelter uns die Welt gegenübertritt - ein zentrales Merkmal der Informationsgesellschaft -, um so schwerer fällt es, diese Erkenntnis und den moralischen Willen, die Konsequenzen daraus zu ziehen, gesellschaftlich zu vermitteln. Der Wald stellt für mich in diesem Sinn einen Präzedenzfall dar, an dem sich die Behandlung der Umwelt in der Informationsgesellschaft studieren läßt.

\section{Anmerkungen}

Es handelt sich um das Projekt "Formen umweltbezogenen Handelns in Situationen von Unsicherheit am Beispiel des Handelns der Schweizer Bevölkerung in bezug auf den Wald", das von der Gruppe Quantitative Geographie/Humanökologie am Geographischen Institut der ETHZ 1989-92 durchgeführt wurde. Der revidierte Schlußbericht erscheint in Buchform (vgl. REICHERT und ZIERHOFER 1993).

${ }^{2}$ Vgl. Bundesgesetz über den Wald vom 4. Oktober 1991, Art. 1.

${ }^{3}$ Alle Interviewpassagen erscheinen unter Pseudonymen und sind sinngetreu gekürzt.

${ }^{4}$ Zur Korrespondenz gesetzlicher und kultureller Waldbegriffe vgl. WALTHER und HUGENTOBLER 1987.

${ }^{5}$ Das Landesforstinventar erhebt alle zehn Jahre vielfältige Informationen über den Zustand des Waldes. Nicht zu verwechseln mit der Waldschadeninventur, die jährlich den Nadel- und Blattverlust erfaßt. 
${ }^{6}$ Zu diesen gehören die Abholzung der Regenwälder und der Verbrauch der Buschwälder semiarider Zonen ebenso wie die Aufforstung von Bannwäldern in den Alpen und die Ausscheidung von Urwaldreservaten im Mittelland.

${ }^{7}$ Vgl. Schweizer Naturschutz, Nr. 5, 1992.

${ }^{8} \mathrm{Vgl}$. BACHMANN 1990.

${ }^{9}$ Vgl. Eidgenössische Forschungsanstalt für Wald, Schnee und Landschaft 1992.

${ }^{10} \mathrm{Vgl}$. z. B. Eidg. Departement des Innern, 1984.

"Vgl. Schweizer Illustrierte, 29.8.1988.

${ }_{12}$ Da die Stichproben geschichtet sind, können keine Signifikanztests durchgeführt werden.

${ }^{13}$ Vgl. GLÜCK 1987.

${ }^{14}$ Für Gegenmaßnahmen.

${ }^{15}$ Zur Versicherung der Beurteilungsgrundlagen.

${ }^{16}$ Um die Politik zu implementieren und langfristig zu stabilisieren.

${ }^{17}$ Zur Berechnung möglicher Folgekosten und zur Evaluation möglicher Maßnahmen.

${ }^{18}$ Via Medien zur Frage von Marketing, Konsum und Lobbying: So geschehen bei der Automobilbranche.

${ }^{19}$ So treibt letztlich auch im Falle des "Waldsterbens" die unsichtbare Hand inr (gutes oder böses?) Spiel.

\section{Literatur}

BACHMANN, P. (1990): Forsteinrichtung und Walderhaltung. ETH Zürich 227, S. 6-8.

Bundesversammlung der Schweizerischen Eidgenossenschaft (1991): Bundesgesetz über den Wald (Waldgesetz, WaG) vom 4. Oktober.

Eidgenössisches Departement des Innern (Hrsg., 1984): Waldsterben und Luftverschmutzung, Bern.

Eidgenössische Forschungsanstalt für Wald, Schnee und Landschaft (Hrsg., 1992): Waldschadenforschung in der Schweiz: Stand der Kenntnisse. Tagungsband zu «Forum für Wissen 1992", Birmensdorf.

GLÜCK,P. (1987): Das Wertesystem der Forstleute. Centralblatt der Gesellschaft für Forstwesen Nr. 1, 104. Jg., S. 44-51.

REICHERT, D., ZIERHOFER,W. (unter Mitarbeit von Bättig, C., Ernste, H., Steiner, D., Vetterli, M. [1993]): Umwelt zur Sprache bringen. Westdeutscher Verlag, Opladen.

Schweizer Illustrierte (1988): Waldsterben: Fehldiagnose? Nr. 35, 29.8., S. 9-15.

Schweizerischer Bund für Naturschutz (1992): Naturwald. Schweizer Naturschutz, Nr. 5.

WALTHER, P., HUGENTOBLER,F. (1987): Wald, bosch.... sprachanalytische Skizzen zu einem Raumplanungsprojekt im Malcantone. Geographica Helvetica 42 (4), S. 266-276. 